\title{
Características da Dinâmica Folicular e Regressão Luteal de Vacas das Raças Gir e Nelore após Tratamento com Cloprostenol Sódico ${ }^{1}$
}

\author{
Álan Maia Borges ${ }^{2}$, Ciro Alexandre Alves Torres ${ }^{3}$, José Reinaldo Mendes Ruas ${ }^{4}$, \\ Vicente Ribeiro Rocha Júnior ${ }^{5}$, Giovanni Ribeiro de Carvalho ${ }^{3}$, Jeferson Ferreira da Fonseca ${ }^{6}$, \\ Alberto Marcatti Neto ${ }^{4}$, Anderson Jorge de Assis ${ }^{7}$
}

\begin{abstract}
RESUMO - A dinâmica folicular e a regressão luteal foram avaliadas em 12 vacas da raça Gir e em sete da raça Nelore tratadas com cloprostenol sódico entre os dias 10 e 12 do ciclo estral. A porcentagem de sincronização foi superior a $92 \%$ para as duas raças, e a ovulação do folículo dominante da segunda onda foi verificada em 72,7\% da raça Gir e em todas as vacas Nelore. O diâmetro do folículo ovulatório na raça Nelore foi inferior $(11,0 \pm 0,9 \mathrm{~mm})$ ao da raça $\operatorname{Gir}(13,0 \pm 1,7 \mathrm{~mm})$. O intervalo médio da aplicação do luteolítico ao estro, à ovulação e o momento de ovulação após o início do estro foram de 88,7 e 91,6h, 119 e 113h, 26,5 e 24,3h para as raças Nelore e Gir, respectivamente.
\end{abstract}

Palavras-chave: bovino, corpo lúteo, luteolítico, sincronização de estro, zebu

\section{Follicular Dynamic and Luteal Regression in Gir and Nelore Cows after Treatment with Cloprostenol Sodic}

\begin{abstract}
The follicular dynamics and luteal regression were studied in 12 Gir and seven Nelore cows, treated with analog prostaglandin $F_{2} \alpha$, between day 10 and 12 of the estrous cycle. The synchronization rate was higher than $92 \%$ for the two breeds, and the ovulation of second dominant follicle occurred in $72.7 \%$ of Gir and all Nelore cows. Ovulatory follicle diameter for Nelore was lower $(11.0 \pm 0.9 \mathrm{~mm})$ than Gir cows $(13.0 \pm 1.7 \mathrm{~mm})$. The average intervals from treatment to estrus, treatment to ovulation time and from the beginning of the estrus to ovulation were 88.7 and 91.6h, 119 and $113 \mathrm{~h}, 26.5$ and $24.3 \mathrm{~h}$ for Nelore and Gir cows, respectively.
\end{abstract}

Key Words: bovine, corpus luteum, estrus synchronization, luteolitic, zebu

\section{Introdução}

Desde que se estabeleceu a ação da prostaglandina $\mathrm{F}_{2} \alpha$ como o hormônio responsável pela regressão do corpo lúteo, vários estudos foram realizados envolvendo o uso desta substância ou análogos para a sincronização do estro, com a finalidade de melhorar a eficiência reprodutiva na espécie bovina. Contudo, existe grande variabilidade nas respostas aos tratamentos, principalmente no que se refere ao intervalo tratamento-estro e à ausência de resposta aos tratamentos iniciados antes do dia sete (Wiltbank, 2000).

O número de trabalhos realizados com acompanhamento da dinâmica folicular e do crescimento e regressão luteal de animais Bos taurus indicus, após aplicação de agente luteolítico, ainda é limitado (Viana et al., 1999a), quando comparado com os de animais Bos taurus taurus.
$\mathrm{O}$ tratamento com $\mathrm{PGF}_{2} \alpha$ ou seus análogos leva à regressão do corpo lúteo e à manifestação de novo estro. Contudo, o intervalo do tratamento à ovulação depende do estádio de desenvolvimento do folículo dominante no momento do tratamento. Se o folículo estiver em sua fase de crescimento ou no início da fase estática, pode-se tornar o folículo ovulatório. Caso contrário, nova onda folicular emergirá, e este intervalo será mais longo. Assim, os tratamentos iniciados em diferentes fases do ciclo estral poderão levar à ovulação do folículo dominante da primeira, segunda ou terceira onda de crescimento folicular (Kastelic et al., 1990).

A luteólise induzida por $\mathrm{PGF}_{2} \alpha$ parece ser mais rápida em zebuínos (Galina et al., 1995), o que faz com que o estro, a onda ovulatória de LH e a ovulação aconteçam mais precocemente em relação ao estro normal (Randel, 1984).

\footnotetext{
1 Parte da tese de Doutorado do primeiro autor, parcialmente financiada pela FAPEMIG.

2 Med.Vet. autônomo-DsC-Rua Cap. Neves ,408. CEP: 37.170-000 B. Esperança-MG. Bolsista do CNPq. E.mail: amborges@hotmail.com

3 Professor do Departamento de Zootecnia da UFV - Av.PH Holfs, s/nº CEP: 36570-000 - Viçosa - MG. E.mail: ctorres@ufv.br; giovanni@ufv.br

${ }^{4}$ Pesquisador da EPAMIG - Viçosa - MG. E.mail: jrmruas@ufv.br

5 Prof. Titular - curso de Zootecnia - Unimontes - Salinas - MG. E.mail: rochajuniorvr@hotmail.com

6 Pesquisador Embrapa Gado de Leite (CNPGL). E.mail: fonsecajef@embrapa.cnpgl.br

7 Aluno do curso de Doutorado da UFV - Viçosa - MG.
} 
A ultra-sonografia tem sido usada, na maioria das vezes, em estudos científicos que caracterizam a dinâmica folicular durante o ciclo estral, proporcionando entendimento das características do desenvolvimento e regressão folicular nos vários estádios fisiológicos. Também pode ser utilizada como ajuda no estudo do mecanismo de regressão do corpo lúteo e o desenvolvimento dos folículos após a aplicação de prostaglandina $\mathrm{PGF}_{2} \alpha$ ou após os tratamentos superovulatórios. Dessa forma, esta técnica permite verificar as variações na resposta aos tratamentos hormonais, como as diferenças nos intervalos da aplicação do agente luteolítico ao início do estro de bovinos, possibilitando assim a escolha do período ideal para iniciar tratamentos.

Este trabalho foi desenvolvido objetivando-se o estudo da regressão luteal, do desenvolvimento folicular e do momento de ovulação de vacas das raças Gir e Nelore tratadas com cloprostenol sódico entre o décimo e o décimo segundo dia do ciclo estral.

\section{Material e Métodos}

O experimento foi conduzido em parceria da Universidade Federal de Viçosa (UFV) e a Empresa de Pesquisa Agropecuária de Minas Gerais (EPAMIG), na Fazenda Experimental de Felixlândia, durante os meses de julho e agosto de 2000.

Foram utilizadas 12 vacas da raça Gir e sete da raça Nelore, de condição corporal boa ou muito boa, com escores 4 a $5(1=$ muito magra, $5=$ muito gorda; citado por Ferreira, 1990), de caracterização racial bem definida, não lactantes e pesos vivos médios de 414 e $459 \mathrm{~kg}$ para as vacas das raças Gir e Nelore, respectivamente. As vacas foram mantidas em um único pasto de topografia plana, formado por capimbraquiária (Brachiaria decumbens e Brachiaria brizantha). Os piquetes possuíam cochos cobertos para sal mineral e bebedouros cimentados para água.

Os animais foram selecionados por meio de exame ginecológico (palpação retal e vaginoscopia) e por estarem ciclando regularmente, sendo utilizados aqueles sem qualquer alteração clínica ou reprodutiva.

A ultra-sonografia foi realizada pela via transretal a partir do dia do estro, utilizando-se um aparelho portátil da marca ALOKA, modelo SSD-500, acoplado a um transdutor linear de $5 \mathrm{MHz}$. Efetuaram-se medições diárias registrando-se os diâmetros máximos do maior e segundo maior folículos presentes em cada ovário, bem como da área da seção transversal do corpo lúteo, segundo metodologia de Ginther et al. (1989). A área do tecido luteal foi calculada pela diferença entre a área da seção transversal do CL e da cavidade luteal. O volume do tecido luteal e da cavidade do corpo lúteo foi calculada pela fórmula matemática: $\mathrm{V}=4 / 3$ P.a/2.(b/2) ${ }^{2}$, em que $\mathrm{a}=$ eixo longitudinal e $\mathrm{b}=$ eixo transversal, modificada de Grygar et al. (1997). A coleta de dados iniciou-se no dia do estro natural e terminou no dia da ovulação do estro induzido. Os valores obtidos foram tabulados para representação gráfica da dinâmica folicular.

Os animais de cada raça foram divididos aleatoriamente em três tratamentos:

$\mathrm{T} 1$ = aplicação de $0,500 \mathrm{mg}$ de cloprostenol sódico ${ }^{1}$ (análogo sintético da $\mathrm{PGF}_{2} \alpha$ ) no décimo dia do ciclo estral (Gir $=5$ e Nelore $=2$ animais $)$

$\mathrm{T} 2$ = aplicação de $0,500 \mathrm{mg}$ de cloprostenol sódico (análogo sintético da $\mathrm{PGF}_{2} \alpha$ ) no décimo primeiro dia do ciclo estral ( $\mathrm{Gir}=3$ e Nelore $=3$ animais $)$

$\mathrm{T} 3$ = aplicação de $0,500 \mathrm{mg}$ de cloprostenol sódico (análogo sintético da $\mathrm{PGF}_{2} \alpha$ ) no décimo segundo dia do ciclo estral ( $\mathrm{Gir}=4 \mathrm{e}$ Nelore $=2$ animais)

Após a aplicação do agente luteolítico, a manifestação de estro foi monitorada visualmente três vezes ao dia: manhã (6 às $7 \mathrm{~h})$, tarde (12 às $13 \mathrm{~h})$ e noite (18 às $19 \mathrm{~h}$ ), com o auxílio de rufião equipado com buçal marcador. Os animais encontrados em estro (marcados ou aceitando monta) foram submetidos à avaliação ultra-sonográfica em intervalos de 6 horas a partir da detecção do estro, até o momento em que o folículo ovulatório desapareceu do ovário. O momento de ovulação (horas) foi considerado como o intervalo médio da última avaliação que o folículo esteve presente ao seu desaparecimento.

A atividade luteal foi monitorada pela determinação da concentração plasmática de progesterona. As amostras de sangue foram coletadas no dia do estro e a cada três dias até o próximo estro, incluindo o dia da aplicação da prostaglandina. Utilizaram-se tubos vacuolizados de $10 \mathrm{~mL}$ contendo solução anticoagulante (EDTA), puncionando a artéria ou veia coccígea. As amostras foram imediatamente centrifugadas a $336,3 \mathrm{~g}$ durante 15 minutos para a separação do plasma, que foi transferido para tubetes plásticos previamente identificados e, então, estocados a $-18^{\circ} \mathrm{C}$. As análises de progesterona foram realizadas no Setor de Reprodução Animal do Departamento de Zootecnia da UFV, por metodologia de radioimunoensaio (RIA) em fase sólida, utilizando-se "Kits" comerciais ${ }^{2}$ e seguindo-se as recomendações

\footnotetext{
R. Bras. Zootec., v.32, n.1, p.85-92, 2003
} 
dos fabricantes.

$\mathrm{Na}$ análise estatística utilizou-se o programa SAEG 8.0, aplicando-se análise de variância para as variáveis foliculares, luteais, para o intervalo prostaglandina-estro e para o momento de ovulação. Para a área e o volume do corpo lúteo, aplicou-se análise de regressão, tendo como fonte de variação as raças e os dias do ciclo estral. As diferenças nas concentrações de progesterona foram testadas por análises de variância e regressão, tendo como fonte de variação os tratamentos e os dias de amostragem. Análise de correlação de Pearson foi feita entre a área, o volume e as concentrações de progesterona.

\section{Resultados e Discussão}

A análise das características avaliadas (foliculares, luteais, intervalo prostaglandina-estro, momento de ovulação e concentrações de progesterona) não mostrou diferença $(\mathrm{P}>0,05)$ entre as vacas das duas raças e os tratamentos T1, T2 e T3 (dia da aplicação da prostaglandina). Tanto nas vacas da raça Gir $(n=8 ; 72,7 \%)$ quanto nas Nelore $(n=7 ; 100 \%)$ houve predominância de ovulações a partir de folículos da segunda onda folicular. Somente um animal Gir ovulou o folículo da primeira onda e, em dois animais, o da terceira onda. Um animal não respondeu ao tratamento com luteolítico (Figura 1).

A administração de $\mathrm{PGF}_{2} \alpha$ entre o $10^{\circ}$ e o $12^{\circ}$ dia do ciclo estral coincidiu com a fase estática ou com o início da regressão do folículo dominante da primeira onda folicular, e com a fase de crescimento dos folículos da segunda onda. A ovulação do folículo dominante da primeira onda foi o resultado da aplicação de $\mathrm{PGF}_{2} \alpha$ no dia 10 , que coincidiu com sua fase de crescimento. No caso de ovulações na terceira onda folicular, $\mathrm{a} \mathrm{PGF}_{2} \alpha$ foi aplicada no $11^{\circ}$ e $12^{\circ}$ dia e coincidiu com o menor período de dominância folicular do FD da segunda onda folicular, que permitiu o surgimento da terceira onda em um intervalo de tempo mais curto (três dias) que o normalmente verificado (nove dias). A emergência precoce de determinada onda folicular pode ser um dos fatores responsáveis pelas falhas na ovulação de outras ondas. Assim, quando os animais são tratados no período em que se espera o surgimento da segunda
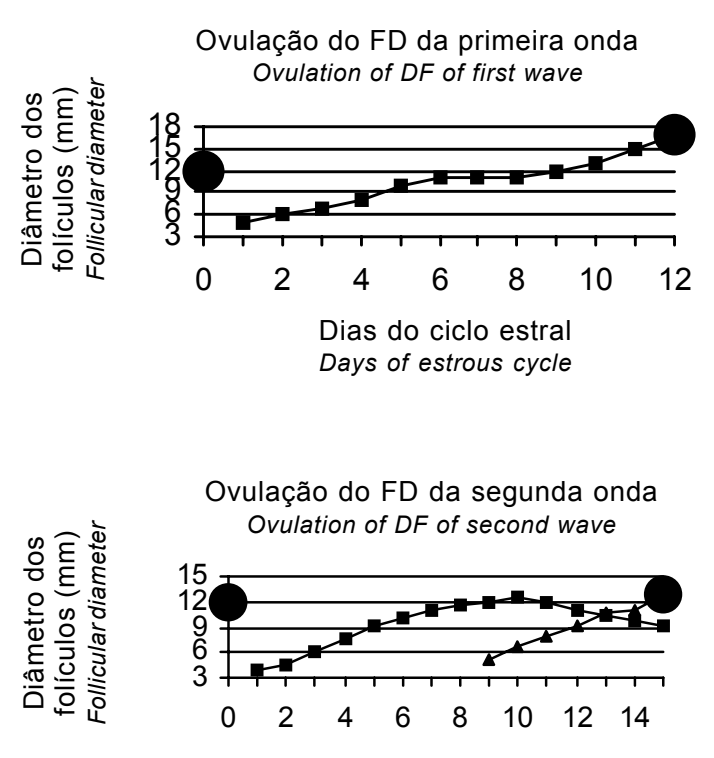

Dias do ciclo estral Days of estrous cycle
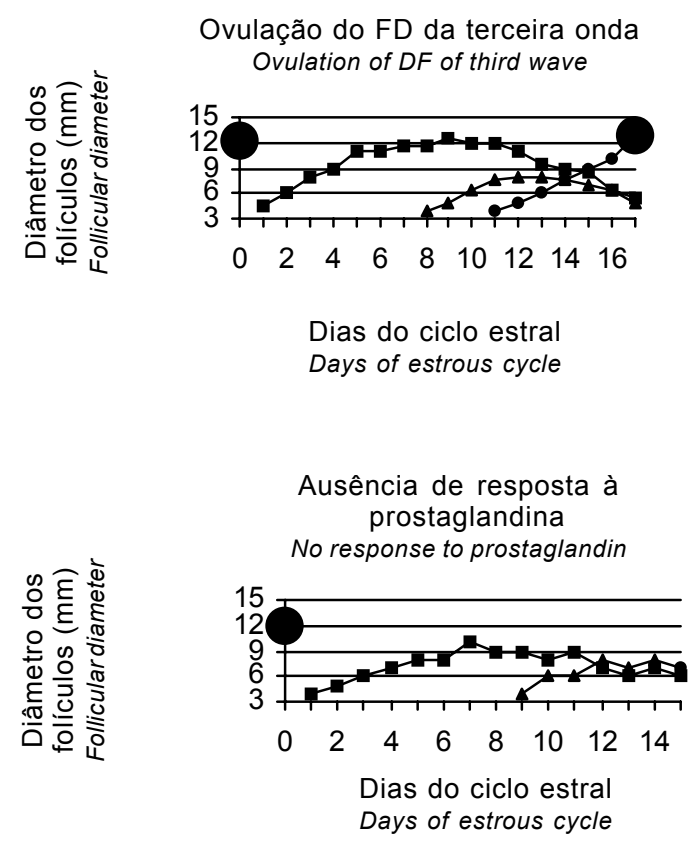

Figura 1 - Desenvolvimento folicular de vacas das raças Gir e Nelore apresentando ovulação do folículo dominante (FD) da primeira $\left(\mathrm{PGF}_{2} \alpha=10^{\circ} \mathrm{dia}\right)$, segunda $\left(\mathrm{PGF}_{2} \alpha=11^{\circ} \mathrm{dia}\right)$ ou terceira $\left(\mathrm{PGF}_{2} \alpha=\right.$ $\left.11,5^{\circ} \mathrm{dia}\right)$ onda folicular. O símbolo de maior tamanho indica o folículo ovulatório.

Figure 1 - Follicular development of Gir and Nelore cows presenting ovulation of the dominant follicle (DF) from first $\left(P G F_{2} \alpha=10^{\text {th }}\right.$ day), second $\left(P G F_{2} \alpha=11^{\text {th }}\right.$ day) or third $\left(P G F_{2} \alpha=11.5^{\text {th }}\right.$ day) follicular wave. The great symbol is the ovulatory follicle.

\footnotetext{
${ }_{1}^{1}$ Ciosin - Mallinckrodt Veterinary - Divisão Coopers do Brasil.

2 Coat-a-count Progesterone - DPC - Diagnostic Products Corporation - Los Angeles - USA.
}

R. Bras. Zootec., v.32, n.1, p.85-92, 2003 
onda folicular (dia 8 a 10), o intervalo do tratamento à ovulação dependerá do estádio de desenvolvimento folicular (Kastelic, 1994).

$\mathrm{O}$ animal que não manifestou estro em resposta ao luteolítico apresentou regressão luteal normal. O folículo da segunda onda atingiu um diâmetro de 8 $\mathrm{mm}$ e persistiu até o final do período experimental. Normalmente, a ausência de resposta deve-se à falha no processo de luteólise (Viana et al., 1999a), que permite a manutenção de concentrações de progesterona semelhante à observada em animais tratados com implantes de progestágeno. Esse animal apresentou regressão luteal normal, o que foi confirmado pela redução na concentração de progesterona.

Onze vacas da raça Gir (92\%) e sete da raça Nelore (100\%) manifestaram estro após o tratamento com prostaglandina. Estes resultados de sincronização foram similares aos encontrados em animais de raças européias (70 a 90\%)(Odde, 1990) e superiores aos de alguns estudos com vacas da raça Nelore, que mostraram resultados próximos a $40 \%$ (Pinheiro et al., 1998). Esta variabilidade nas respostas está em função do dia em que os tratamentos foram iniciados. A variabilidade no período de ocorrência do estro após o uso da $\mathrm{PGF}_{2} \alpha$ deve-se às diferenças no crescimento folicular entre as vacas, e não no tempo de regressão do corpo lúteo (Wiltbank, 2000). A aplicação de luteolítico nos primeiros cinco dias do ciclo estral coincide com o período de desenvolvimento do corpo lúteo, que não é totalmente responsivo à prostaglandina. $\mathrm{O}$ tratamento entre os dias cinco e nove pode levar à luteólise parcial e os iniciados entre 11 e 15 dias, os melhores resultados (Odde, 1990). A ausência de resposta aos tratamentos iniciados na presença de um corpo lúteo pouco responsivo pode ser contornada pela aplicação de uma segunda dose de luteolítico após 10 a 11 dias (novilhas) e de 13 a 14 dias para as vacas em lactação após a primeira aplicação (Wiltbank, 2000).

A baixa porcentagem de detecção de estros em animais zebuínos após os tratamentos com luteolítico (30 a 40\%) também pode ser atribuída às falhas na identificação, tanto pela falta de habilidade do responsável quanto pela carência de um período suficiente de observação, o que compromete os resultados dos programas de inseminação artificial (Galina et al., 1987).

No dia da aplicação da $\mathrm{PGF}_{2} \alpha$, o diâmetro médio do folículo dominante foi de 7,3 $\pm 2,4 \mathrm{~mm}$. Este folículo cresceu 3,5 dias e atingiu o diâmetro ovulatório de 13,0 $\pm 1,7 \mathrm{~mm}$ para a raça Gir e de $11,0 \pm 0,9 \mathrm{~mm}$ para a raça Nelore. Houve diferença $(\mathrm{P}<0,05)$ no diâmetro do FD à ovulação para as duas raças, devido à sua menor taxa de crescimento dos FD de vacas da raça Nelore em relação à raça Gir (Tabela 1).

$\mathrm{O}$ intervalo da aplicação de $\mathrm{PGF}_{2} \alpha$ à manifestação do estro não variou entre as raças e nem com os diferentes dias da aplicação do luteolítico $(\mathrm{P}>0,05)$ (Tabela 2).

Verifica-se na literatura que o intervalo da aplicação do luteolítico à deteç̧ão do estro é bastante variável tanto para zebuínos quanto taurinos. O intervalo encontrado nesse estudo $(88,7$ a $91,6 \mathrm{~h})$ foi similar às $91,6 \mathrm{~h}$ em animais zebuínos de corte (Hardin et al., 1980). Tem sido encontrado valores de 53,4h para vacas da raça Nelore (Valle et al., 1994), 63,5h para a raça Indubrasil (Vaca et al., 1985), 59,9h para a raça Gir e de 64,6h para a raça Guzerá (Alves, 2001). Estas diferenças podem ser devidas às diferenças na metodologia utilizada na detecção de estros, quando realizadas em intervalos de seis horas ou de forma ininterrupta, já que os estros iniciados no período noturno seriam identificados somente na observação da manhã, no sistema tradicional de observação.

Tabela 1 - Parâmetros foliculares de vacas das raças Gir e Nelore sincronizadas com prostaglandina $F_{2} \alpha$ Table 1 - Follicular parameters of Gir and Nelore cows synchronized with prostaglandin $F_{2} \alpha$

\begin{tabular}{|c|c|c|}
\hline $\begin{array}{l}\text { Característica } \\
\text { Characteristic }\end{array}$ & Gir & Nelore \\
\hline $\begin{array}{l}\text { Taxa de crescimento } \\
\text { anterior à } \mathrm{PGF}_{2} \alpha(\mathrm{mm} / \mathrm{dia}) \\
\text { Growth rate before the } P G F_{2} \alpha\end{array}$ & $1,3 \pm 0,8 \mathrm{a}$ & $1,1 \pm 0,6 \mathrm{a}$ \\
\hline $\begin{array}{l}\text { Taxa de crescimento } \\
\text { após a } \mathrm{PGF}_{2} \alpha(\mathrm{mm} / \mathrm{dia}) \\
\text { Growth rate after the } P G F_{2} \alpha\end{array}$ & $1,6 \pm 0,3 \mathrm{a}$ & $1,2 \pm 0,2 b$ \\
\hline $\begin{array}{l}\text { Número de dias de crescimento } \\
\text { anterior à } \mathrm{PGF}_{2} \alpha \\
\text { Number of growth days } \\
\text { before the } \mathrm{PGF}_{2} \alpha\end{array}$ & $2,7 \pm 2,3 \mathrm{a}$ & $2,2 \pm 1,6 a$ \\
\hline $\begin{array}{l}\text { Número de dias de crescimento } \\
\text { após a } \mathrm{PGF}_{2} \alpha \\
\text { Number of growth days after the } P\end{array}$ & $\begin{array}{l}3,2 \pm 1,6 \mathrm{a} \\
F_{2} \alpha\end{array}$ & $3,7 \pm 1,4 a$ \\
\hline
\end{tabular}


Tabela 2 - Médias e desvios-padrão para o intervalo (horas) da aplicação da prostaglandina ao início do estro e à ovulação de vacas das raças Gir e Nelore

Table 2 - Mean and standard error for the interval (hours) between the prostaglandin treatment to beginning of estrous and to ovulation in Gir and Nelore cows

\begin{tabular}{lcc}
\hline Raça & $\mathrm{PF}_{2} \alpha$ ao estro & $\mathrm{PF}_{2} \alpha$ à ovulação \\
Breed & $P G F_{2} \alpha$ to estrous & $P G F_{2} \alpha$ to ovulation \\
& Média \pm desvio & Média \pm desvio \\
\hline
\end{tabular}

Gir $\quad 91,6 \pm 28,0 \mathrm{~h}(\mathrm{n}=11) \mathrm{A} \quad 113,1 \pm 30,2 \mathrm{~h}(\mathrm{n}=11) \mathrm{B}$

Nelore $\quad 88,7 \pm 26,1 \mathrm{~h}(\mathrm{n}=7) \quad 119,5 \pm 31,9 \mathrm{~h}(\mathrm{n}=7) \mathrm{B}$

Valores seguidos por letras maiúsculas distintas na mesma coluna diferem entre si $(P<0,05)$ pelo teste $F$.

Values with different capital letters within column differ $(P<.05)$ by $F$ test.

O intervalo médio do tratamento ao estro foi mais curto naqueles animais que ovularam o FD da primeira onda (dois dias) em comparação àqueles ovulando o FD da segunda (dois a cinco dias) e terceira ondas (cinco a sete dias). Os intervalos do tratamento com luteolítico ao estro e à ovulação foram maiores e mais variáveis quando iniciados por volta do dia 10 do ciclo estral em relação ao início mais cedo ou mais tarde (Kastelic, 1994). O comprimento deste intervalo depende da viabilidade do folículo dominante ao início do tratamento.

A avaliação ultra-sonográfica freqüente pode indicar os momentos apropriados ou não para iniciar os processos de sincronização estral com prostaglandina. Caso seja verificado o surgimento de uma nova onda folicular, é de se esperar maior intervalo do tratamento à ovulação, quando comparado com a detecção de um FD no final de sua fase de crescimento ou no início da fase estática (Bo et al., 1994). Entretanto, a identificação do folículo dominante apenas por sua caracterização morfológica (folículo $>9 \mathrm{~mm}$ de diâmetro) pode incorrer em erros, quando feita apenas uma única avaliação ultrasonográfica. Isto porque, nem sempre, o maior folículo presente em dado momento é funcionalmente dominante. Somente dois animais $(10,5 \%)$ ovularam o maior folículo presente ao momento de aplicação da prostaglandina. Os demais $(n=17 ; 89,5 \%)$, que apresentavam o maior folículo na fase estática ou em regressão, não responderam com ovulação.

Os melhores resultados de sincronização do estro são obtidos quando a prostaglandina é aplicada no momento que o folículo dominante está na fase de crescimento ou início da fase estática, e quando coincidem com a regressão luteal (Kastelic et al.,
1994). Os corpos lúteos, quando funcionais, apresentam elevada secreção de progesterona - hormônio que faz "feedback" negativo ao eixo hipotalâmicohipofisário-, impedindo a onda de LH, que é responsável pela maturação final do folículo e pela ovulação (Adams et al., 1992).

A ovulação ocorreu, em média, de 113,1 $\pm 30,2 \mathrm{~h}$ a $119,5 \pm 31,9$ h após a aplicação de $\mathrm{PGF}_{2} \alpha$. Foram encontrados valores de 114h (Moreno et al., 1986), em vacas Indubrasil e de 93 a 97 horas para as raças Gir e Guzerá (Alves, 2001). Nota-se a amplitude das variações citadas na literatura, que, segundo MacMillan \& Henderson (1984), é devida à sincronização do momento da luteólise, mas não do estádio de desenvolvimento dos folículos dominantes. A sincronização de estro após o uso da $\mathrm{PGF}_{2} \alpha$ não é suficientemente precisa para proporcionar bons resultados com a IA programada tanto para o gado zebu (Cavalieri et al., 1997) quanto nos de raças européias (Pursley et al., 1997), quando comparada com a observação visual do estro (Wright \& Malmo, 1992).

Segundo Galina et al. (1995), é possível que, em zebuínos, a luteólise induzida por $\mathrm{PGF}_{2} \alpha$ seja mais rápida, fazendo com que o estro, a onda ovulatória de LH e a ovulação aconteçam mais precocemente em relação ao estro normal (Randel, 1984).

O intervalo do início do estro induzidoà ovulação não variou entre as raças $(\mathrm{P}>0,05)$ e foi, em média, de 24,3 $\pm 6,2$ horas para as vacas Gir e de 26,5 $\pm 3,5$ horas para as vacas Nelore. Intervalos de 29 e $28 \mathrm{~h}$ para as vacas Gir e Nelore, respectivamente, foram encontrados por Alves (2001) e Pinheiro et al. (1998) encontraram 26,7 horas para as vacas Nelore. Estes resultados mostraram valores similares com o momento de ovulação de estros naturais, em que foram encontrados intervalos de 28h para animais das raças Gir (Viana et al., 1999b), $26,8 \mathrm{~h}$ para a Nelore (Pinheiro et al., 1998) e $32 \mathrm{~h}$ nas vacas mestiças (Wettemann et al., 1998). As variações no momento de ovulação podem ser decorrentes da técnica utilizada para sua determinação.

O desenvolvimento luteal entre o estro natural e o induzido, bem como as equações de regressão que representam sua área e volume, é demonstrado na Figura 2. A área, o volume e as concentrações de progesterona não variaram entre as raças $(\mathrm{P}>0,05)$, mas foram diferentes para os dias do ciclo estral $(\mathrm{P}<0,01)$.

A área e volume inicial à detecção do corpo lúteo foram, respectivamente, de $1,9 \mathrm{~cm}^{2}$ e $1,8 \mathrm{~cm}^{3}$, sendo primeiramente identificados ao dia 2,9 . O corpo lúteo cresceu à taxa de $0,3 \mathrm{~cm}^{2} /$ dia até o dia 6,8 a 7,1 , 
quando atingiu sua maior área $\left(2,5 \mathrm{a} 2,7 \mathrm{~cm}^{2}\right) \mathrm{e}$ volume $\left(2,8 \mathrm{a} 2,9 \mathrm{~cm}^{3}\right)$, calculados pelo ponto de máxima. No dia da aplicação do agente luteolítico, a área média foi de 3,2 $\mathrm{cm}^{2}$. Essas variações decorrem da formação e do desenvolvimento do corpo lúteo a partir do tecido remanescente do processo ovulatório. Posteriormente, a redução progressiva nas medidas luteais foi devida à aplicação do agente luteolítico, que induziu modificações morfofisiológicas luteais, por meio da redução na vascularização tecidual e morte celular por apoptose (McCracken et al., 1999). A regressão do corpo lúteo ocorreu em 3,9 $\pm 1,0$ dias a partir do tratamento com o luteolítico, a uma taxa de $0,6 \mathrm{~cm}^{2} /$ dia.

Paralelamente ao desenvolvimento e à regressão luteal, as concentrações plasmáticas de progesterona aumentam e declinam bruscamente neste mesmo período (Vaca et al., 1983). Os maiores valores de progesterona foram obtidos entre os dias 11,8 e 11,9 para as raças Gir e Nelore, respectivamente, calculados pelo ponto de máxima, quando atingiram valores máximos entre 3,7 e 4,6 ng/mL. As concentrações de progesterona refletem a funcionalidade luteal entre sua fase de crescimento, manutenção e regressão, de modo que os maiores valores foram encontrados por volta do dia 10, igual ao encontrado por Wise et al. (1982).

As correlações de Pearson entre a área, o volume e as concentrações de progesterona mostraram-se positivas e altas (Tabela 3). À medida que o corpo lúteo se desenvolve, as concentrações de progesterona aumentam proporcionalmente, e sua regressão é acompanhada pela menor secreção de progesterona. $\begin{array}{ll}\text { Gir: } \hat{Y}=0,017+0,775 \mathrm{D}-0,055 \mathrm{D}^{2} & \mathrm{R}^{2}=0,91(\mathrm{P}<0,01) \\ \text { Nelore: } \hat{Y}=0,393+0,612 \mathrm{D}-0,045 \mathrm{D}^{2} & \mathrm{R}^{2}=0,97(\mathrm{P}<0,01)\end{array}$

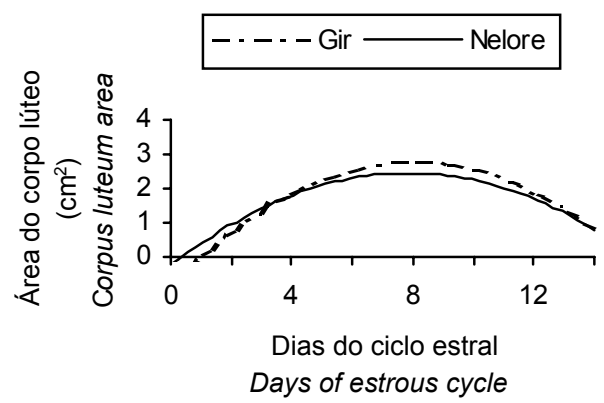

$\hat{Y}=1,080-1,077 \mathrm{D}+0,258 \mathrm{D}^{2}-0,012 \mathrm{D}^{3}$

$R^{2}=1,0(P<0,01)$

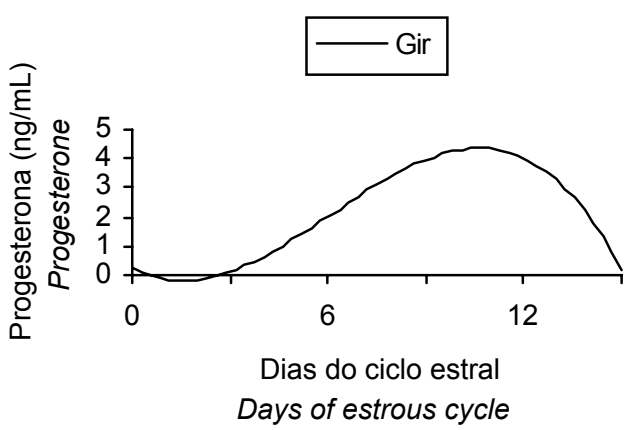

Gir: $\hat{Y}=-0,662+1,005 \mathrm{D}-0,071 \mathrm{D}^{2}$

$R^{2}=0,85(P<0,01)$

Nelore: $\hat{Y}=-0,328+0,913 D-0,066 D^{2}$

$R^{2}=0,95(P<0,01)$

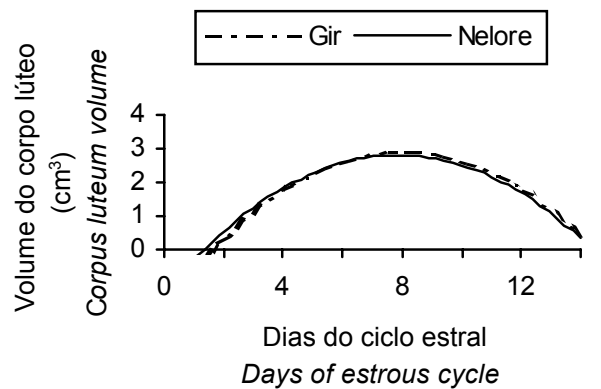

$\hat{Y}=0,225-0,259 \mathrm{D}+0,118 \mathrm{D}^{2}-0,006 \mathrm{D}^{3} \quad \mathrm{R}^{2}=0,95(\mathrm{P}<0,01)$

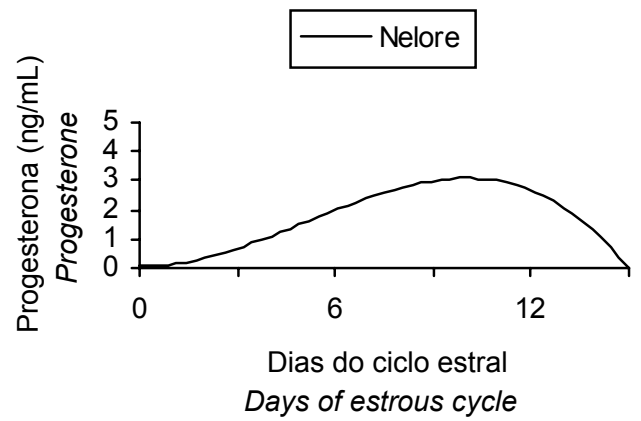

Figura 2 - Área e volume dos corpos lúteos, e concentrações plasmáticas de progesterona de vacas das raças Gir e Nelore, em função dos dias do ciclo estral.

Figure 2 - Area and volume of the corpus luteum and plasma progesterone concentration in Gir and Nelore cows, by the day of estrous cycle.

R. Bras. Zootec., v.32, n.1, p.85-92, 2003 
Tabela 3 - Correlações simples de Pearson entre a área e o volume do corpo lúteo com as concentrações plasmáticas de progesterona de vacas das raças Gir e Nelore sincronizadas com prostaglandina $F_{2} \alpha$, em função dos dias do ciclo estral

Table 3 - Simple Pearson correlations between area and volume of corpus luteum with plasma progesterone concentration, in Gir and Nelore cows synchronized with prostaglandin $-F_{2} \alpha$

\begin{tabular}{|c|c|c|c|c|}
\hline \multirow{2}{*}{$\begin{array}{l}\text { Variáveis } \\
\text { Variables }\end{array}$} & \multicolumn{2}{|c|}{ Gir } & \multicolumn{2}{|c|}{ Nelore } \\
\hline & $\begin{array}{l}\text { Correlação } \\
\text { Correlation } \\
\end{array}$ & $\begin{array}{c}\text { Significância } \\
\text { Significance }\end{array}$ & $\begin{array}{l}\text { Correlação } \\
\text { Correlation } \\
\end{array}$ & $\begin{array}{c}\text { Significância } \\
\text { Significance }\end{array}$ \\
\hline $\begin{array}{l}\text { Área } x \text { Volume } \\
\text { Area } x \text { volume }\end{array}$ & 0,98 & $\mathrm{P}<0,01$ & 0,97 & $\mathrm{P}<0,01$ \\
\hline $\begin{array}{l}\text { Área x Progesterona } \\
\text { Area x progesterone }\end{array}$ & 0,73 & $\mathrm{P}<0,01$ & 0,74 & $\mathrm{P}<0,01$ \\
\hline $\begin{array}{l}\text { Volume } \mathrm{x} \text { Progesterona } \\
\text { Volume } x \text { progesterone }\end{array}$ & 0,74 & $\mathrm{P}<0,01$ & 0,73 & $\mathrm{P}<0,01$ \\
\hline
\end{tabular}

\section{Conclusões}

O tratamento com cloprostenol sódico (análogo sintético da $\mathrm{PGF}_{2} \alpha$ ) entre o décimo e o décimo segundo dia foi eficiente na indução de estros em vacas das raças Gir e Nelore.

As respostas ao cloprostenol sódico dependem da condição folicular no momento do tratamento, o que se comprova pelo fato de o folículo dominante funcional tornar-se ovulatório, quando o tratamento com cloprostenol sódico induz a luteólise.

O intervalo do tratamento à ovulação é função da fase de dinâmica folicular, quando da aplicação do luteolítico, e depende da normalidade funcional do folículo dominante.

\section{Literatura Citada}

ADAMS, G.P.; MATTERI, R.L.; KASTELIC, J.P. et al. Association between surges of FSH and emergence of follicular waves in heifers. Journal Reproduction and Fertility, v.94, p.177-188, 1992.

ALVES, N.G. Estação do ano e tipo de luteólise sobre as características do proestro e estro de vacas das raças Gir e Guzerá (Bos taurus indicus). Belo Horizonte: Universidade Federal de Minas Gerais, 2001. 93p. Dissertação (Mestrado em Medicina Veterinária) - Universidade Federal de Minas Gerais, 2001.

BO, G.A.; ADAMS, G.P.; PIERSON, R.A. et al. Follicular wave dynamics after estradiol-17 $\beta$ treatment of heifers with or without a progestagen implant. Theriogenology, v.41, p.1555-1569, 1994

CAVALIERI, J.; RUBIO, I.; KINDER, J.E. et al. Synchronization of estrus and ovulation and associated endocrine changes in Bos indicus cows. Theriogenology, v.47, p.801-814, 1997.

FERREIRA, A.M. Efeito da amamentação e do nível nutricional na atividade ovariana de vacas mestiças leiteiras. Viçosa, MG: Universidade Federal de Viçosa, 1990. 134p. Tese (Doutorado em Zootecnia) - Universidade Federal de Viçosa, 1990

GALINA, C.S.; ORIHUELA, A.; DUCHATEAU, A. Reproductive physiology in zebu cattle - Unique reproductive aspects that affect their performance. Veterinary Clinical of North American - Food Animal Practice, v.3, n.3, p.619-632, 1987.

GALINA, C.S.; ORIHUELA, A.; RUBIO, I. Reproductive physiology in zebu cattle, characteristics related to estrus expression and performance of bulls utilized in natural mating. IN: CONGRESSO BRASILEIRO DE REPRODUÇÃO ANIMAL, 9., 1995, Belo Horizonte. Anais... Belo Horizonte: Colégio Brasileiro de Reprodução Animal, 1995. p.46-61.

GINTHER, O.J.; KNOPF, L.; KASTELIC, J.P. Temporal associations among ovarian events in cattle during oestrus cycles with two and three follicular waves. Journal Reproduction and Fertility, v.87, p.223-230, 1989.

GRYGAR, I.; KUDLÁC, E.; DOLEZEF, R. et al. Volume of luteal tissue and concentration of serum progesterone in cows bearing homogeneous corpus luteum or corpus luteum cavity. Animal Reproduction Science, v.49, p.77-82, 1997.

HARDIN, D.R.; WARNICK, A.C.; FIELDS, M.J. Artificial insemination of subtropical commercial beef cattle following synchronization with cloprostenol (ICI 80996): II. Estrous response. Theriogenology, v.14, n.4, p.259-268, 1980.

KASTELIC, J.P. Understanding ovarian follicular development in cattle. Veterinary Medicine, v.6, p.64-71, 1994.

MacMILLAN, K.L.; HENDERSON, H.V. Analysis of the variation in the interval from injection of prostaglandin $\mathrm{F}_{2} \alpha$ to estrous as a method of studying patterns of follicle development during dioestrus in dairy cows. Animal Reproduction Science, v.6, p.245-254, 1984.

McCRACKEN, J.A.; CUSTER, E.E.; LAMSA, J.C. Luteolysis: A neuroendocrine-mediated event. Physiological Reviews, v.79, n.2, p.263-324, 1999.

MORENO, I.Y.D.; GALINA, C.S.; ESCOBAR, F.J. et al. Evaluation of the lytic response of prostaglandin $\mathrm{F}_{2}$ alpha in zebu cattle based on serum progesterone. Theriogenology, v.25, n.3, p.413-421, 1986.

ODDE, K.G. A review of synchronization of estrus in postpartum cattle. Journal Animal Science, v.68, p.817-830, 1990.

PINHEIRO, O.L.; BARROS, C.M.; FIGUEIREDO, R.A. et al.

R. Bras. Zootec., v.32, n.1, p.85-92, 2003 
Estrous behaviour and the estrus-to-ovulation interval in Nelore cattle (Bos indicus) with natural estrus or estrus induced with prostaglandin $\mathrm{F}_{2} \alpha$ or Norgestomet and estradiol valerate. Theriogenology, v.49, n.3, p.667-681, 1998.

PURSLEY, J.R.; WILTBANK, M.C.; STEVENSON, J.S. et al. Pregnancy rates in cows and heifers inseminated at a synchronized ovulation or synchronization estrus. Journal Dairy Science, v.80, p.295-300, 1997.

RANDEL, R.D. Seasonal effects on female reproductive functions in the bovine (Indian breeds). Theriogenology, v.21, p.170-185, 1984.

VACA, L.A.; GALINA, C.; FERNÁNDEZ-BACA, S. et al. Progesterone levels and relationship with the diagnosis of a corpus luteum by rectal palpation during the estrous cycle in zebu cows. Theriogenology, v.20, n.1, p.67-76, 1983.

VACA, L.A.; GALINA, C.S.; FERNÁNDEZ-BACA, S. Oestrous cycles, oestrus and ovulation of the zebu in the Mexican tropics. Veterinary Review, v.117, n.17, p.434-437, 1985.

VALLE, E.R.; ENCARNAÇÃO, R.O.; SCHENK, J.A.P. et al. Duração do cio e momento de ovulação em vacas Nelore. Revista da Sociedade Brasileira de Zootecnia, v.23, n.5, p.852-858, 1994.

VIANA, J.H.M.; PIRES, M.F.A.; CAMARGO, L.S.A. et al. Dinâmica folicular no período pré-ovulatório, estro e momento de ovulação em vacas Gir. Revista Brasileira de Reprodução Animal, v.23, n.3, p.159-160, 1999b.
VIANA, J.H.M; FERREIRA, A.M.; SÁ, W.F. et al. Regressão luteal e dinâmica folicular após luteólise natural ou induzida por cloprostenol em vacas da raça Gir. Arquivo Brasileiro de Medicina Veterinária e Zootecnia, v.51, n.3, p.257-262, 1999a.

WETTEMANN, R.P.; LOOPER, M.L.; WARNER, M.L. et al. Estrous behavior and time of ovulation in beef cows during the summer. Animal Science Research Report, p.227-231, 1998.

WILTBANK, M.C. Uso eficaz de hormônios de reprodução: II. Programas de reprodução. In: NOVOS ENFOQUES NA PRODUÇÃO E REPRODUÇÃO DE BOVINOS, 4., 2000, Passos. Anais... Passos: 2000. p.71-85.

WISE, T.H.; CATON, D.; THATCHER, W.W. et al. Ovarian function during the estrous cycle of the cow: ovarian blood flow and progesterone release rate. Journal of Animal Science, v.55, n.3, p.627-637, 1982.

WRIGHT, P.J.; MALMO, J. Pharmacologic manipulation of fertility. Veterinary Clinical Of North American: Food Animal Practice, v.8, n.1, p.57-89, 1992.

Recebido em: 10/04/02

Aceito em: 30/08/02 